\title{
Efecto de la distribución de tamaño de partícula de AIN empleado como refuerzo durante la fabricación de materiales compuestos de Mg-AZ91E por infiltración sin presión externa
}

\section{Effect of particle size distribution of AIN used as reinforcement during the fabrication of Mg-AZ91E composites by infiltration without external pressure}

\author{
DOI: 10.46814/lajdv3n6-001
}

Recebimento dos originais: 01/10/2021

Aceitação para publicação: 09/11/2021

\section{José Lemus-Ruiz \\ Doctor}

Instituto de Investigación en Metalurgia y Materiales, Universidad Michoacana de San Nicolás de Hidalgo, Fco. J. Mújica S/N, Edif. U, C.U., Apdo. Postal 888, C.P. 58000, Morelia, Mich., México.

E-mail: jlruiz@umich.mx

\section{RESUMEN}

El presente trabajo se enfoca en la síntesis y caracterización de un material compuesto de matriz de magnesio AZ91E con alto contenido de partículas de refuerzo ( $50 \%$ vol.) de nitruro de aluminio (AlN). La fabricación se realizó por el método de infiltración sin usar presión externa, partiendo de preformas de AlN en verde, es decir sin pre-sinterizar. Las preformas con porosidades de $50 \%$ vol., fueron producidas empleando polvo de AlN de tres distribuciones de tamaño de partícula (A:fina, B:media y C:gruesa) distintas, e infiltradas con la aleación de magnesio AZ91E líquida a $850^{\circ} \mathrm{C}$ durante 12 minutos en una atmósfera de argón. Técnicas de microscopía electrónica de barrido (MEB), así como difracción de rayos $\mathrm{X}$ (DR-X) fueron empleadas para caracterizar los compuestos AZ91E/AlN producidos, evaluando la distribución del refuerzo, porosidad y productos de reacción. AlN, Mg y $\mathrm{Mg}_{17} \mathrm{Al}_{12}$ fueron dtectados por XRD. Los resultados obtenidos por MEB muestran una distribución homogénea del refuerzo, libres de productos de reacción, entre la matriz y el refuerzo, para los tres casos analizados. Resultados de la caracterización mecánica indican valores promedio del módulo de elasticidad alrededor de $123 \mathrm{GPa}$. El coeficiente de expansión térmica lineal oscila en $\approx 9.61 \times 10^{-6 \circ} \mathrm{C}^{-1}$ y $8.96 \times 10^{-6 \circ} \mathrm{C}^{-1}$ en el rango de temperatura entre 25 y $300^{\circ} \mathrm{C}$. La conductividad térmica se incrementa de $47 \mathrm{~W} / \mathrm{mK}$ para el compuesto fabricado con partículas C:gruesas, a $53 \mathrm{~W} / \mathrm{mK}$ para la partículas de tamaño B:medio y a $81 \mathrm{~W} / \mathrm{mK}$ con tamaño de partícula A:fina, medidos a temperatura ambiente, $25^{\circ} \mathrm{C}$, sin embargo la conductividad disminuye para los tres compuestos cuando se incrementa la temperatura de medición.

Palabras Clave: nitruro de aluminio, Mg-AZ91E, infiltración, conductividad térmica.

\begin{abstract}
This work focuses on processing and characterization of metal matrix composites of magnesium alloy AZ91E reinforced with high amount ( $50 \mathrm{vol} . \%)$ of aluminum nitride (AlN). Fabrication was made by the infiltration technique without external pressure, starting of green AlN preforms, without presintered. Preforms with porosities around 50 vol\% were produced with three diferents particle size distributions powders (A:fine, B:medium and C:thick and infiltrated with molted AZ91E magnesium alloy at $850^{\circ} \mathrm{C}$ during 12 minutes in argn atmosphere. The produced AZ91E/AlN composites were characterized through scanning (SEM) as well as X ray diffraction (XRD), reinforcement distribution, porosity, and reaction products were evaluated. SEM results show homogeneous distribution of
\end{abstract}


reinforcement within the matrix, free of reactions products, between the matrix and the reinforcement for all three materials. AlN, $\mathrm{Mg}$ and $\mathrm{Mg}_{17} \mathrm{Al}_{12}$ were detected by XRD. Results of the mechanical and thermal characterization indicate average values of elastic modulus of $123 \mathrm{GPa}$. The lineal thermal expansion coefficient vary between $\approx 9.61 \times 10^{-6 \circ} \mathrm{C}^{-1}$ and $8.96 \times 10^{-6}{ }^{\circ} \mathrm{C}^{-1}$ in the range of temperature between 25 and $300^{\circ} \mathrm{C}$. The thermal conductivity increase of $47 \mathrm{~W} / \mathrm{mK}$ to composited produced xith C:thick particles to $53 \mathrm{~W} / \mathrm{mK}$ to B:midium particles and to $81 \mathrm{~W} / \mathrm{mK}$ with A:fine particles evaluated at room temperatura, $25 \mathrm{C}$, however termal conductivity decrease for the three composites when evaluation temperatura increase.

Keywords: aluminium nitride, Mg-AZ91E, infiltration, thermal conductivity

\section{INTRODUCCIÓN}

Los materiales compuestos están formados por una fase continua llamada matriz, puede ser metálica, cerámica u orgánica, así como una fase de refuerzo dispersa en la matriz y puede ser en forma de fibra o partículas [1] y se obtienen al unir en forma macroscópica dos materiales para conseguir una combinación de propiedades que no es posible obtener en los materiales originales. Generalmente los componentes son significativamente distintos en propiedades, siendo uno ligero, fuerte y frágil y el otro suele ser tenaz y dúctil. Los combinaciones en materiales compuestos pueden ser metal/metal, metal/cerámico, metal/polímero, cerámico/polímero, cerámico/cerámico o polímero/polímero $(1,2]$. La unión de estos materiales disímiles (matriz y refuerzo) dará lugar a materiales con propiedades poco usuales como: rigidez, resistencia, densidad, rendimiento a elevada temperatura, resistencia a la corrosión, dureza, o conductividad eléctrica y térmica. El uso de partículas como refuerzo tiene una mayor acogida en los materiales compuestos de matriz metálica (CMM), ya que asocian menores costos y permiten obtener una mayor isotropía de propiedades en el producto. Sin embargo, para tener éxito en el desarrollo de CMM se debe tener un control estricto del tamaño y la pureza de las partículas utilizadas. Los refuerzos típicos de mayor uso en forma de partículas son: carburos (TiC, B 4 C), óxidos $\left(\mathrm{SiO}_{2}, \mathrm{TiO}_{2}, \mathrm{ZrO}_{2}, \mathrm{MgO}\right)$ y nitruros $\left(\mathrm{AlN}, \mathrm{Si}_{3} \mathrm{~N}_{4}\right)$. Sin embargo, en los últimos años se han empezado a utilizar partículas de intermetálicos, principalmente de los sistemas Ni-Al y Fe-Al [3, 4]. Se ha reportado en la literatura la elaboración de CMM usando como refuerzo AIN utilizando diferentes rutas de procesamiento [5-7]. La densidad del Mg es alrededor de (2/3) de la del Al, (1/4) de la del Zn y (1/5) de la del acero. Como resultado las aleaciones de magnesio ofrecen una resistencia específica alta comparada con las aleaciones convencionales de aluminio, además éstas aleaciones tienen buena capacidad de amortiguamiento, excelente colada y muy buena maquinabilidad [8]. Por otro lado, el AlN poli-cristalino tiene una conductividad térmica de 80 a $200 \mathrm{Kw} / \mathrm{m} . \mathrm{K}$, un coeficiente de expansión térmica de $4.4 \times 10^{-6}{ }^{\circ} \mathrm{C}^{-1}$ (valor muy cercano al del carburo de silicio $3.2 \times 10^{-6}{ }^{\circ} \mathrm{C}^{-1}$ ). Estas dos propiedades hacen al AlN un excelente material para circuitos eléctricos de alta densidad comparado 
con otros sustratos cerámicos que por lo general tienen coeficientes de conductividad térmica bajos y coeficientes de expansión térmica altos. Como resultado, la combinación de las propiedades mecánicas del AlN con las del Mg y sus aleaciones dan origen a un compuesto muy atractivo para aplicaciones electrónicas y estructurales.

Las propiedades de los compuestos están determinadas por factores, tales como las propiedades individuales de los materiales que constituyen la matriz y el refuerzo, así como de su interacción interfacial, además de la morfología, orientación y distribución de la fase reforzarte y de la técnica de fabricación [9]. La reacción interfacial entre la matriz y el refuerzo requiere de un estudio riguroso, ya que puede cambiar la composición de la matriz y el refuerzo, no obstante alguna de estas reacciones interfaciales contribuyen al aumento de las propiedades mecánicas, otras lo harán en detrimento [10], por lo tanto su análisis resulta ser de gran importancia. Una interfase adherida fuertemente es un requisito para una buena resistencia en un compuesto. La naturaleza y calidad de la interfase (morfología, composición química, resistencia y adhesión) son determinadas por factores intrínsecos, tanto del material de refuerzo como de la matriz (composición química, cristalografía y contenido de defectos), así como también factores extrínsecos (tiempo, temperatura, presión y atmósfera) relacionados con la fabricación del material. En los CMM una cantidad moderada de interacciones químicas entre el refuerzo y la matriz mejoran la mojabilidad y la resistencia de la interfase. Tales interacciones frecuentemente resultan en la formación de fases intermedias que no están en equilibrio, debido a que las interfaces son termodinámicamente inestables y las transformaciones morfológicas y estructurales continúan durante todo el proceso de fabricación. Sin embargo, una reacción química excesiva degrada el refuerzo y la resistencia del compuesto $[11,12]$. La naturaleza de la técnica en la fabricación de materiales compuestos tiene un efecto marcado sobre las propiedades del material. En el caso de procesamiento en estado líquido para fabricación de materiales compuestos, la mayor dificultad que se presenta es la no mojabilidad de la fase cerámica por los metales líquidos. Por lo tanto, es de gran importancia obtener una buena mojabilidad para lograr una unión fuerte entre la matriz y el refuerzo $[13,14]$. La infiltración por capilaridad sin presión externa de preformas cerámicas es una técnica atractiva para la fabricación de materiales compuestos, debido a que permite manufacturar materiales con alto contenido de cerámico. Sin embargo, las temperaturas altas que se requieren para fabricar el compuesto por esta técnica, pueden generar una cantidad considerable de productos de reacción en la interfase. El objetivo de este trabajo fue elaborar un material compuesto usando como matriz la aleación de magnesio AZ91E y como refuerzo partículas cerámicas de AlN de tres distribuciones de tamaño distintas, utilizando la técnica de infiltración por capilaridad sin el uso de presión externa. 


\section{METODOLOGÍA EXPERIMENTAL}

La composición química de la aleación de Mg-AZ91E (Thomson Aluminum Casting Co. USA) usada en la fabricación del material compuesto en \% peso es: Mg:90, Al:8.1-9.3, Zn:0.4-1, Si:0.20 $\max , \mathrm{Fe}: 0.005 \max , \mathrm{Cu}: 0.015 \max$ y Ni:0.001 max. El material de refuerzo utilizado fue polvo de AlN (Aldrich Chemical Co. USA) de tres distribuciones de tamaño de partícula denominados como polvos: A:fina, B:media y C:gruesa. La fabricación del compuesto se realizó mediante la técnica de infiltración sin presión externa, para lo cual se fabricaron preformados de los distintos polvos de AlN, sin uso de ligantes, con dimensiones de $6.5 \times 1 \times 1 \mathrm{~cm}$ y pastillas de $1.2 \mathrm{~cm}$ de diámetro y $0.5 \mathrm{~cm}$ de espesor, en dados de acero, prensando uniaxialmente a $15 \mathrm{MPa}$. Las preformas en verde de AlN se colocaron en contacto con trozos pequeños de la aleación Mg-AZ91E dentro de un crisol de grafito en un horno tubular horizontal a una temperatura de $850^{\circ} \mathrm{C}$ durante 12 minutos en atmósfera de argón, como se muestra en el esquema de la Figura 1. Una vez que la aleación se funde, esta se infiltra en el preformado obteniendo así el material compuesto. A los compuestos producidos se les evaluó la densidad y porosidad residual de acuerdo a la norma ASTM C20-00. Así mismo se caracterizaron microestructuralmente por medio de difracción de rayos X en un difractómetro Siemmens D5000 en un rango de barrido de 15 a 80 grados, así mismo se examinó la sección transversal de los compuestos, previa preparación de pulido, usando un microscopio electrónico de barrido JEOL JMS-6400 para analizar las características estructurales y morfológicas tanto del compuesto, como de los polvos de refuerzo de AlN.

Figura 1. Representación esquemática del proceso de infiltración con aleación de Mg-AZ91E de las preformas de AlN

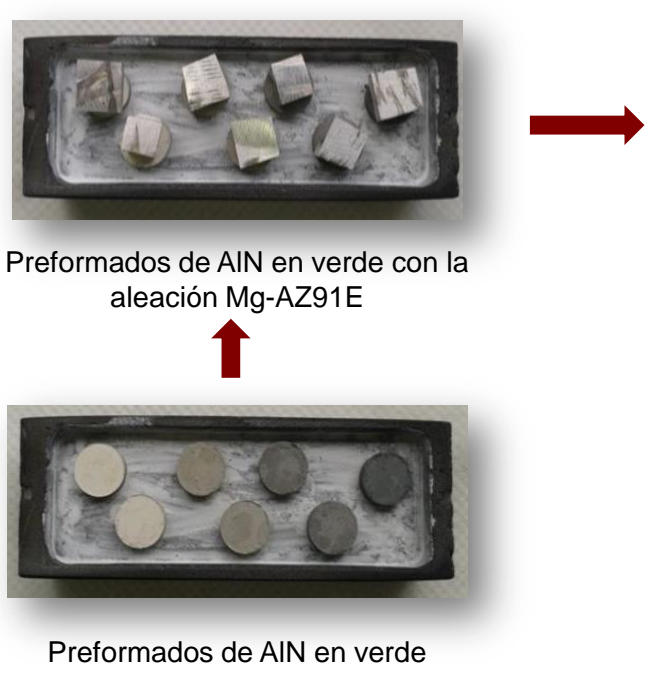

Preformados de AIN en verde

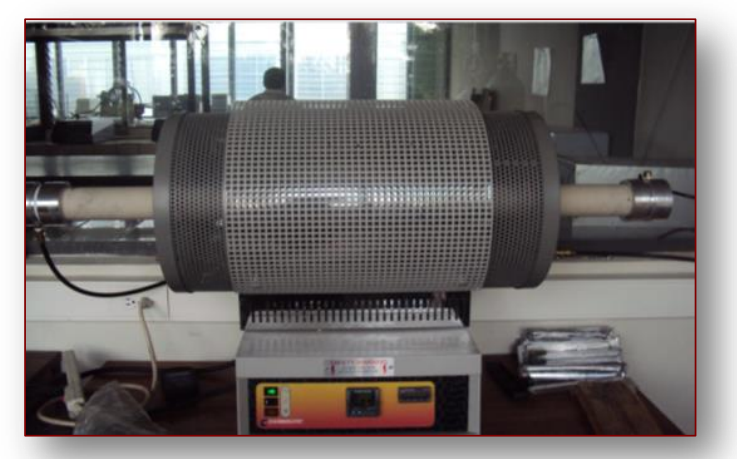

Horno tubular (Infiltración a $850^{\circ} \mathrm{C}$ por 12 min en argón)

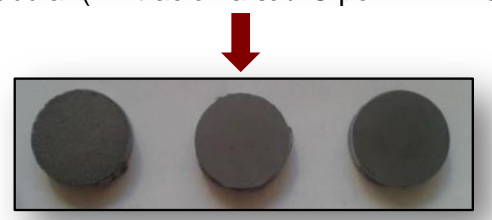

Compuestos AIN/Mg-AZ91E 
Las pruebas para determinar el coeficiente de expansión térmica se realizaron en un dilatómetro vertical Linseis $\mathrm{L} 75$ en atmósfera de nitrógeno en el rango de temperatura entre 25 y $300^{\circ} \mathrm{C}$. La conductividad térmica se midió en un equipo Linseis LFA 1000 en el rango de 25 a $300^{\circ} \mathrm{C}$. La caracterización mecánica consistió en la evaluación del módulo de elasticidad por medio de un equipo Grindo Sonic MK5 JV Lemmens, para lo cual se prepararon 3 barras del compuesto Mg-AZ91E/AlN para cada distribución de tamaño de partícula, A:fina, B:media y C:gruesa, a las cuales se les realizó la medición en cada una de las 4 caras para obtener un valor promedio. Por otro lado, se evaluó de manera teórica el módulo de elasticidad por medio de la ecuación de las mezclas y la ecuación de Halpin Tsai [15].

\section{RESULTADOS Y DISCUSIÓN}

\subsection{MATERIALES DE INICIO}

Para iniciar con la producción de los materiales compuestos de matriz de magnesio AZ91E reforzados con AlN de tres distintos tamaño de partícula denominados como A: fina, B: medio y C: grueso se caracterizaron los materiales de inicio. La figura 2a presenta una imagen de la estructura de la aleación AZ91E donde podemos distinguir la base metálica de $\mathrm{Mg}$ y la fase $\mathrm{Mg}_{17} \mathrm{Al}_{12}$ que es un precipitado común en este tipo de aleación de Mg-AZ91E, la presencia de estas fases se corroboraron con difracción de rayos x. Así mismo, en la figura 2 b y 2c observamos la distribución de tamaño de partícula y la morfología de los polvos de AlN, respectivamente. Podemos observar que en caso de los polvos A: finos y B: medio, presentan una distribución de tamaño de partícula bimodal, predominando los polvos finos en la muestra A y polvos entre las $10 \mu \mathrm{m}$ en la muestra B, sin embargo en el caso de los polvos C: grueso la distribución se concentra en tamaños alrededor de los $17 \mu \mathrm{m}$. En la figura 2c observamos la morfología irregular de los polvos de AlN.

Figura 2. a) aleación Mg-AZ91E, b) distribución de tamaño de partícula, c) morfología de los polvos de AlN empleados en la producción de los compuestos Mg-AZ91E/AlN

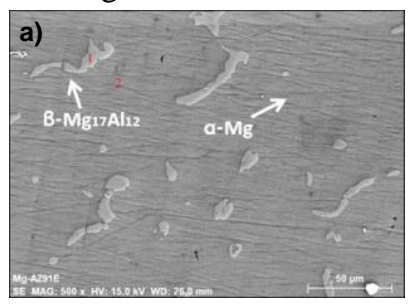

b)

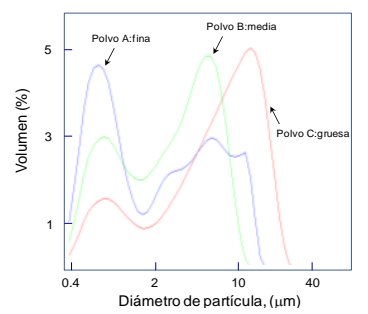

c)
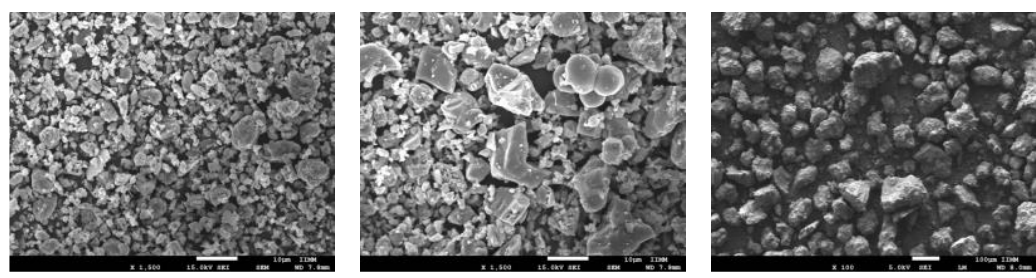


\subsection{ANÁLISIS TERMODINÁMICO}

En acorde a la termodinámica es factible la formación de varios productos de reacción durante la fabricación del compuesto $\mathrm{AZ} 91 \mathrm{E} / \mathrm{AlN}$ a $900^{\circ} \mathrm{C}$. La formación de $\mathrm{MgO}$ y de la espinela $\mathrm{MgAl}_{2} \mathrm{O}_{4}$ puede darse a la temperatura de infiltración por las siguientes reacciones (1) y (2):

$$
\begin{gathered}
\mathrm{Mg}_{(\mathrm{l})}+1 / 2 \mathrm{O}_{2(\mathrm{~g})}=\mathrm{MgO}_{(\mathrm{s})} \\
\Delta \mathrm{G}_{\left(900^{\circ} \mathrm{C}\right)}=-473 \mathrm{~kJ} \\
3 \mathrm{Mg}_{(\mathrm{ll}}+4 \mathrm{Al}_{2} \mathrm{O}_{3(\mathrm{~s})}=3 \mathrm{MgAl}_{2} \mathrm{O}_{4(\mathrm{~s})}+2 \mathrm{Al} \\
\Delta \mathrm{G}_{\left(900^{\circ} \mathrm{C}\right)}=-256 \mathrm{~kJ}
\end{gathered}
$$

La formación de la espinela $\mathrm{MgAl}_{2} \mathrm{O}_{4}$ puede darse aún en estado sólido de acuerdo a la siguiente reacción (3):

$$
\begin{gathered}
\mathrm{MgO}_{(\mathrm{s})}+\mathrm{Al}_{2} \mathrm{O}_{3(\mathrm{~s})}=\mathrm{MgAl}_{2} \mathrm{O}_{4(\mathrm{~s})} \\
\Delta \mathrm{G}_{\left(900^{\circ} \mathrm{C}\right)}=-44 \mathrm{~kJ}
\end{gathered}
$$

Por otro lado, termodinámicamente el $\mathrm{MgO}$ es más estable que la $\mathrm{Al}_{2} \mathrm{O}_{3}$, por tanto, el $\mathrm{Mg}$ puede reducir la $\mathrm{Al}_{2} \mathrm{O}_{3}$ de acuerdo a la siguiente reacción (4):

$$
\begin{gathered}
3 \mathrm{Mg}_{(\mathrm{l})}+\mathrm{Al}_{2} \mathrm{O}_{3(\mathrm{~s})}=3 \mathrm{MgO}_{(\mathrm{s})}+2 \mathrm{Al} \\
\Delta \mathrm{G}_{\left(900^{\circ} \mathrm{C}\right)}=-123 \mathrm{~kJ}
\end{gathered}
$$

Como puede observarse termodinámicamente las reacciones (2) y (3) que dan origen a la formación de espinelas son factibles de ocurrir, sin embargo, el grado de reacción para que se lleve a cabo una u otra dependerá de la temperatura, el tiempo de exposición (cinética) y la composición química de la aleación. $\mathrm{El}_{\mathrm{Al}_{2} \mathrm{O}_{3}}$ es termodinámicamente estable en contacto con $\mathrm{Al}$ puro, sin embargo, tratándose de una aleación de $\mathrm{Mg}$ tiende a formarse $\mathrm{MgO}$ de acuerdo a las ecuaciones (1) y (4) o la espinela $\mathrm{MgAl}_{2} \mathrm{O}_{4}$ de acuerdo a las ecuaciones (2) y (3) y en un sistema que se tenga suficiente oxígeno esta espinela puede formarse de acuerdo a la siguiente reacción (5):

$$
\begin{aligned}
\mathrm{Mg}_{(\mathrm{l})}+2 \mathrm{Al}_{(\mathrm{l})}+2 \mathrm{O}_{2(\mathrm{~g})} & =\mathrm{MgAl}_{2} \mathrm{O}_{4(\mathrm{~s})} \\
\Delta \mathrm{G}_{\left(900^{\circ} \mathrm{C}\right)} & =-1808 \mathrm{~kJ}
\end{aligned}
$$

De acuerdo a investigaciones realizadas por: Lloyd [17], McLeod y col. [18], Lloyd y col. [19], contenidos de $\mathrm{Mg}$ altos y temperaturas bajas favorecen la formación del $\mathrm{MgO}$, mientras que la formación de la espinela es más factible a contenidos de $\mathrm{Mg}$ bajos, misma que en nuestro sistema AZ91E/AlN no fue detectada. 


\subsection{CARACTERIZACIÓN DE LOS MATERIALES COMPUESTOS MG-AZ91E/ALN}

La Figura 3 muestra secciones transversales para los materiales compuestos Mg-AZ91E/AlN para los tres compuestos producidos por la infiltración de $\mathrm{Mg}$-AZ91E a $850^{\circ} \mathrm{C}$ por 12 minutos en preformas porosas con distribución de tamaño de partícula a) fina, b) media y c) gruesa. En la Figura 3 se observa una distribución homogénea en los tres casos de compuestos producidos, con aproximadamente $51 \%$ de la aleación AZ91E y un 49\% de AlN. Las densidades de los compuestos producidos evaluadas por el método de Arquímedes fueron de: $2.56,2.58$ y $2.55 \mathrm{~g} / \mathrm{cm}^{3}$ para el compuesto A:fina, B:media y C:gruesa, respectivamente, recordemos que la fracción del contenido de refuerzo debido a la distribución de tamaño de partículas de los polvos de AlN afecta el tamaño de los poros en el compacto en verde, observándose una mayor compactación en distribución de polvos finos comparada con los polvos gruesos. Esto se observa en la porosidad residual en el compuesto ya que en compactos producidos con partículas gruesas presentan una porosidad de $7 \%$ y en los finos de $10 \%$ asociado en la mayor apertura o tamaño de poros benéfica para la infiltración.

Figura 3. Compuestos $\mathrm{Mg}$-AZ91E/AlN infiltrados a $850^{\circ} \mathrm{C}$ por 12 minutos para polvos de AlN con distribuciones de tamaño de partícula a) A:fina, b) B:media y c) C:gruesa

a)

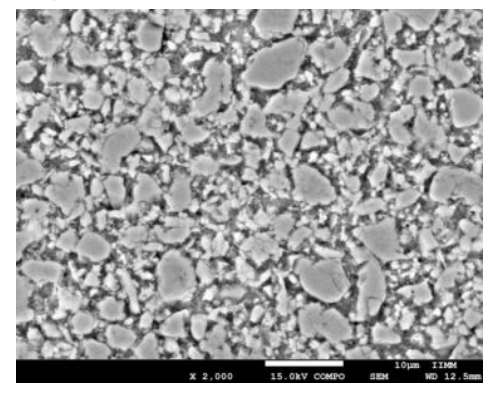

b)

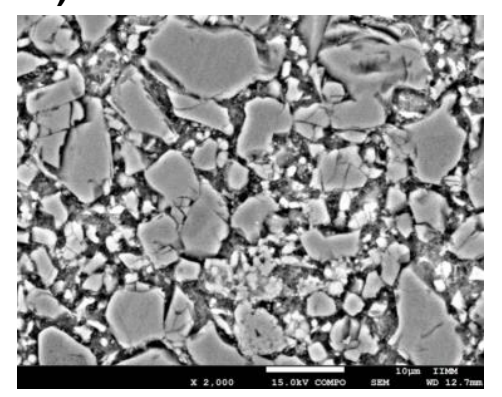

c)

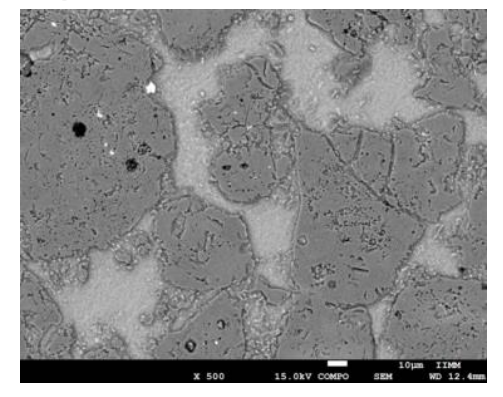

Acorde a la termodinámica es factible la formación de varios productos de reacción durante la fabricación del compuesto $\mathrm{Mg}-\mathrm{AZ} 91 \mathrm{E} / \mathrm{AlN}$ a $850^{\circ} \mathrm{C}$. Las observaciones en las muestras de los compuestos realizadas en MEB a altas magnificaciones muestran que aparentemente no existen productos de reacción en la interfase. Sin embargo, para corroborar la ausencia de reacción entre la matriz y el refuerzo es necesario realizar un estudio de las muestras por microscopía electrónica de transmisión. Contreras y col. [16] realizaron un estudio de la interfase metal/cerámico en estos compuestos pero producidos con preformados presinterizados a $1550^{\circ} \mathrm{C}$ previos a la infiltración a $900^{\circ} \mathrm{C}$, por microscopia electrónica de transmisión y observaron la formación de otras fases como el $\mathrm{MgO}$ y la espinela $\mathrm{MgAl}_{2} \mathrm{O}_{4}$. Sin embargo en nuestro caso estas fases no fueron detectadas por medio de la técnica de DR-X, lo cual puede atribuirse a la poca cantidad de productos de reacción que se podría formar. 
El perfil cuantitativo de componentes a través de la línea dentro del compuesto producido con distribución tamaño de partícula de AlN denominada B:media, se presenta en la Figura 4. La línea donde se realiza el análisis pasa por distintas regiones de la matriz Mg-AZ91E y de partículas de refuerzo de AlN. Podemos observar en la Figura 4 como el contenido de los componentes cambia de acuerdo a la región en la que nos encontramos, así el perfil del Al y N se incrementa al máximo nivel cuando estamos dentro de una partícula de AlN y disminuye cuando estamos en la matriz. Perfil opuesto al observado en el $\mathrm{Mg}$, el cual alcanza su máximo valor cuando estamos pasando por las regiones de la matriz.

Figura 4. Análisis en línea para el compuesto con distribución de tamaño de partícula de AlN, B:media, infiltrado a $850^{\circ} \mathrm{C}$ por 12 minutos.
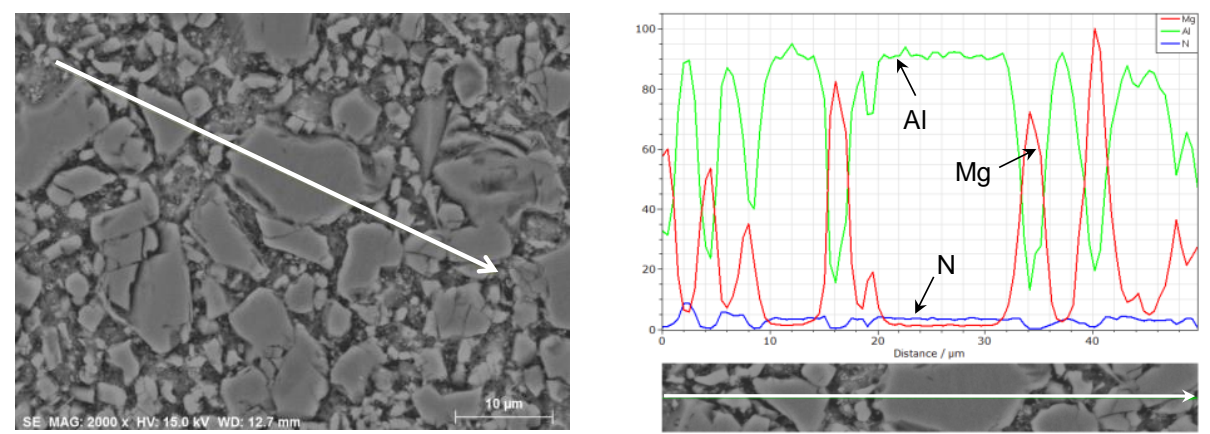

Así mismo, con el propósito de clarificar la distribución de los componentes de la matriz de Mg-AZ91E y del refuerzo de AlN dentro del material compuesto, la Figura 5 presenta los resultados para la distribución atómica de los componentes $\mathrm{Mg}$, Al y $\mathrm{N}$ para el compuesto infiltrado en la muestra con polvos gruesos.

Figura 5. Distribución atómica en compuestos con distribuciones de tamaño de partícula de AlN con polvos C:gruesos infiltrados a $850^{\circ} \mathrm{C}$ por 12 minutos.
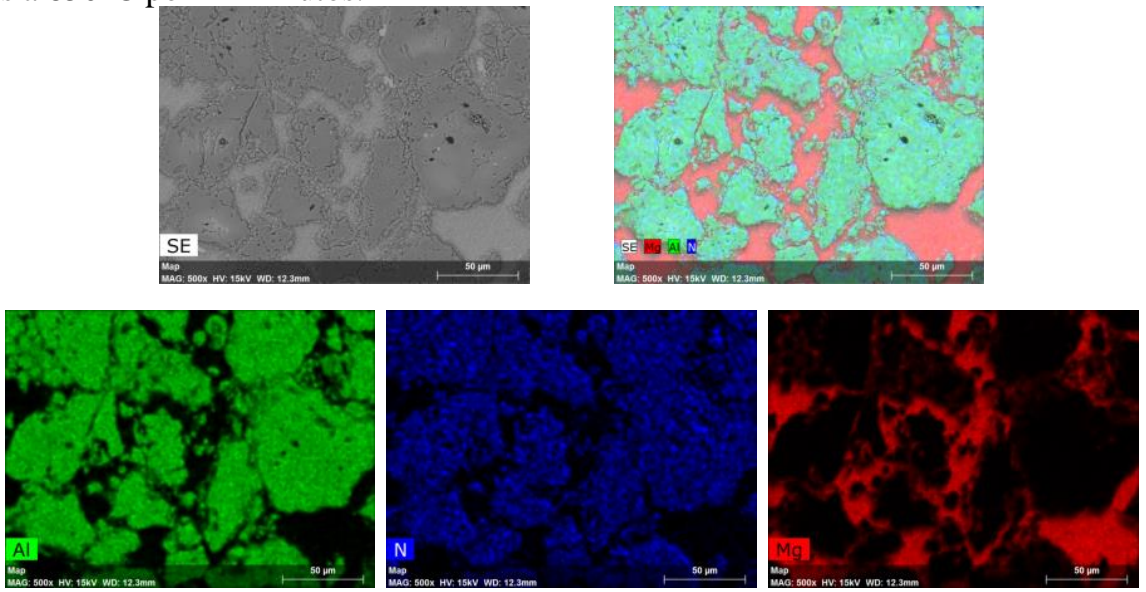
Empleando está técnica de distribución atómica o mapeo, es factible apreciar en forma cualitativa las regiones donde se encuentran los componentes del compuesto, clarificando la buena distribución en forma dispersa de las partículas de AlN dentro de la matriz continua de Mg. En la etapa de preparación de las muestras para su análisis por microscopia electrónica de barrido no se detectó desprendimiento de partículas en ninguno de los tres tipos de compuestos producidos, por lo que se puede decir que existe una buena adhesión entre el cerámico y la matriz.

\subsection{CARACTERIZACIÓN MECÁNICA Y TÉRMICA DE LOS COMPUESTOS}

Para evaluar el grado de adhesión entre refuerzo y matriz se utiliza el trabajo de adhesión, el cual es una medida que refleja el grado de unión entre la superficie sólida y líquida. Una interfase adherida fuertemente es un requisito para tener un buen comportamiento mecánico y estructural en un compuesto. La naturaleza y calidad de la interfase (morfología, composición química, resistencia y adhesión) son determinadas por factores intrínsecos tanto del material de refuerzo como de la matriz (composición química, cristalografía y contenido de defectos) así como también por factores intrínsecos relacionados con el proceso de fabricación (tiempo, temperatura, presión, atmósfera, entre otros). A los compuestos Mg-AZ91E/AlN fabricados con diferente distribución de tamaño de partícula: A:fina, B:media y C:gruesa, se les evaluaron algunas propiedades mecánicas como es el módulo de elasticidad, ya que está directamente relacionadas con el volumen de refuerzo en la matriz metálica. Los resultados del módulo de elasticidad evaluado en el material por medio del equipo Grindo Sonic se muestran en la Figura 6. Como se puede observar el valor del módulo elástico no presenta un cambio marcado entre los tres distintos materiales compuestos (108 a $122 \mathrm{GPa}$ ), sin embargo es marcadamente superior al valor de la matriz monolítica de Mg-AZ91E (44 GPa), atribuido al refuerzo de AlN de los materiales compuestos.

Figura 6. Módulo de Young para los compuestos con distribución de tamaño de partícula de AlN: A:fina, B:media y C:gruesa infiltrados a $850^{\circ} \mathrm{C}$ por 12 minutos.

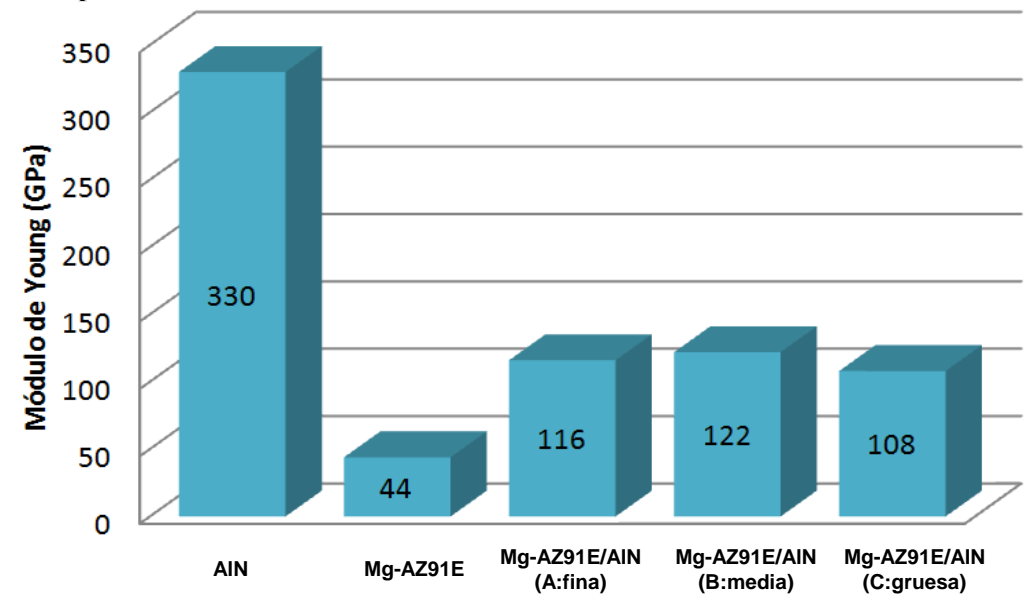


El valor del módulo de elasticidad obtenido en los compuestos Mg-AZ91E/AlN fabricados en este trabajo son ligeramente menores a los encontrados en el estudio reportado por Lai y Chung [20] quienes fabricaron un compuesto de $\mathrm{Al} / \mathrm{AlN}$ con $58 \%$ de refuerzo, obteniendo un módulo de elasticidad de $144 \mathrm{GPa}$, sin embargo, el porcentaje de refuerzo que ellos usaron fue mayor al que aquí se reporta y el módulo de elasticidad del aluminio es mayor que el de la aleación AZ91E. así mismo el valor del módulo calculado empleando el modelo de Halpin Tsai está en 150MPa.

Por otro lado, los materiales compuestos pueden ser empleados como materiales estructurales o funcionales, dependiendo de las propiedades que presentan. De este modo, el compuesto AZ91E/AlN podría ser utilizado como material de empaquetamiento electrónico, aplicación que requiere materiales con coeficientes de expansión térmica (CET) baja y conductividad térmica alta, debido a la baja deformación dimensional y elevada disipación del calor generado durante su aplicación. Los resultados para la conductividad térmica media para los tres compuestos fabricados, medida a las temperaturas de $25,100,200$ y $300^{\circ} \mathrm{C}$ se presentan en la Figura 7. Podemos observar que la conductividad térmica se reduce con el incremento de la temperatura de evaluación, sin embargo la conductividad térmica se incrementa cuando la distribución de tamaño de partícula se incrementa, así pasa de $47 \mathrm{~W} / \mathrm{mK}$ para el compuesto fabricado con partículas C:gruesa, a $53 \mathrm{~W} / \mathrm{mK}$ para la partículas de tamaño B:medio y a 81 $\mathrm{W} / \mathrm{mK}$ con tamaño de partícula A:fina, medidos a temperatura ambiente, $25^{\circ} \mathrm{C}$. El cambio en la conductividad térmica puede asociarse a la diferente conectividad de las partículas cerámicas de refuerzo dentro de la matriz de Mg-AZ91E y tener diferente distribución de tamaño de partícula.

Figura 7. Conductividad térmica medida a distintas temperaturas para los compuestos con distribución de tamaño de partícula de AlN: A:fina, B:media y C:gruesa infiltrados a $850^{\circ} \mathrm{C}$ por 12 minutos.

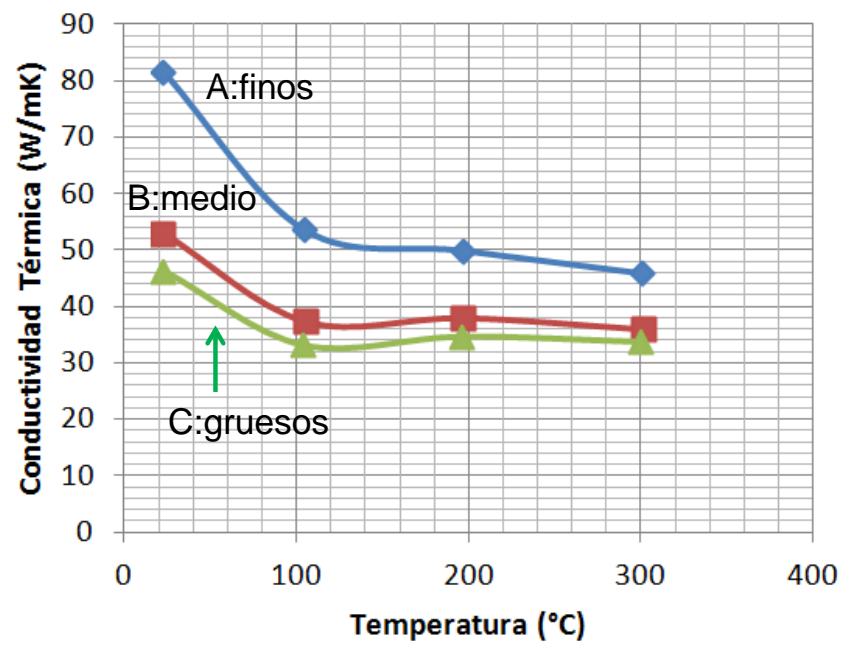


Por otro lado, los compuestos Mg-AZ91E/AIN fabricados, presentan un CET bajo que varía de $9.61 \times 10^{-6 \circ} \mathrm{C}^{-1}$ para el compuesto A:fina, $9.21 \times 10^{-6 \circ} \mathrm{C}^{-1}$ para el compuesto B:media y de $8.963 \times 10^{-6 \circ} \mathrm{C}^{-}$ ${ }^{1}$ para el C:gruesa, en el rango de temperatura entre 25 a $300^{\circ} \mathrm{C}$. Zhang y col. [21] reportan valores de $11.2 \times 10^{-6 \circ} \mathrm{C}^{-1}$ para un compuesto de $\mathrm{Al} / \mathrm{AlN}$ con $50 \%$ de refuerzo, así mismo, Lai y Chung [20] reportan un valor de $10.16 \times 10^{-6}{ }^{\circ} \mathrm{C}^{-1}$ para un compuesto de $\mathrm{Al} / \mathrm{AlN}$ con $54.6 \%$ de refuerzo.

\section{CONCLUSIONES}

En el presente trabajo se llevó a cabo la fabricación y caracterización de un material compuesto Mg-AZ91E/AlN empleando tres polvos de AlN con distribución de tamaño de partícula, empleando la técnica de infiltración sin presión externa. Preformas en verde, es decir sin previa pre-sinterización, fueron infiltradas con la aleación Mg-AZ91E a $850^{\circ} \mathrm{C}$. Los estudios de MEB muestran que el refuerzo y la matriz están homogéneamente distribuidos sin la formación aparente de una interfase o bien sin productos de reacción entre la matriz y el refuerzo. Resultados de la caracterización mecánica indican valores promedio del módulo de elasticidad cercanos entre los tres compuestos con una variación entre 108 a $122 \mathrm{GPa}$. El coeficiente de expansión térmica lineal es de $9.61 \times 10^{-6 \circ} \mathrm{C}^{-1}$ y $8.96 \times 10^{-6 \circ} \mathrm{C}^{-1}$ en el rango de temperatura entre 25 a $300^{\circ} \mathrm{C}$ y la conductividad térmica a $25^{\circ} \mathrm{C}$ disminuye en el orden de 81 , 53 y $47 \mathrm{~W} / \mathrm{mK}$ para los compuestos con distribución de tamaño de partícula A:fina, B:media y C:gruesa, respectivamente, sin embargo disminuye para los tres casos cuando se incrementa la temperatura de medición.

\section{AGRADECIMIENTOS}

Los autores agradecen el apoyo recibido por parte del Consejo Nacional de Ciencia y Tecnología (CONACYT-Proyecto: 167286) y a la Coordinación de la Investigación Científica de la Universidad Michoacana de San Nicolás de Hidalgo. 


\section{REFERENCIAS}

1. D.R. Askeland, "Ciencia e Ingeniería de los Materiales", 3ra Edición, Thomson International Thomson. España, 1998.

2. A. Contreras-Cuevas, E. Bedolla-Becerril, M. Salazar-Martínez, J. Lemus-Ruiz, "Metal Matrix Composites: Wetting and Infiltration", $1^{\text {st }}$ Edition, Springer International Publishing, Springer Nature Switzerland AG, 2018. DOI:10.1007/978-3-319-91854-9

3. C.E. Da Costa, F.V. López, J.M.Y. Castello, "Materiales compuestos de matriz metálica: Parte I. Tipos, propiedades, aplicaciones", Revista de Metalurgia, Vol. 36, No. 3, p. 179-192, 2000. DOI:10.3989/revmetalm

4. Y. Sahin, M. Acilar, "Production and properties of SiCp-reinforced aluminium alloy composites". Composites: Part A, Vol. 34, p. 709-718, 2003. DOI:10.1016/S1359-835X(03)00142-8

5. H.Z. Ye, X.Y. Liu, B. Luan, "In-situ synthesis of AlN in Mg-Al alloy by liquid nitridation", J. Mater. Proc. Tech., Vol. 166, p. 79-85, 2005. DOI:10.1016/j.jmatprotec.2004.06.033

6. S. Swaminathan, B. Srinivasa Rao, V. Jayaram, "The production of AlN-rich matrix composites by the reactive infiltration of Al alloys in nitrogen", Acta Mater., Vol. 50, p. 3093-3104, 2002. DOI:10.1016/s1359-6454(02)00103-9

7. D.-F. Liia, J.-L. Huang, S.-T. Chang, "The mechanical properties of AlN/Al composite fabricated by squeeze casting", J. Eur. Cer. Soc., Vol. 22, p. 253-261, 2002. DOI: 10.1016/S09552219(01)00255-2

8. H.Z. Ye, X.Y. Liu, "Review of recent studies in magnesium matrix composites, J. Mater. Sci., Vol. 39, p. 6153-6171, 2004. DOI: 10.1023/B:JMSC.0000043583.47148.31

9. A. Contreras, C. A. León, R.A.L. Drew, E. Bedolla, "Wettability and spreading kinetics of Al and Mg on TiC", Scrip. Mater., Vol. 48, p. 1625-1630, 2003. DOI: 10.1016/S1359-6462(03)00137-4

10. D. Muscat, K. Shanker, R.A.L. Drew, "Al/TiC composites produced by melt infiltration", Mat. Sci.\& Tech., Vol. 8, p. 971-976, 1992. DOI: 10.1179/mst.1992.8.11.971

11. R. Asthana, "Reinforced cast metal part I: Solidification and microstructure", J. Mater. Sci., Vol. 33, No. 7, p. 1679-1698, 1998.

12. R. Asthana, "Reinforced cast metal part II: Evolution of interface", J. Mater. Sci., Vol. 33, No. 8, p. 1959-1980, 1998. DOI: 10.1023/A:1004334228105

13. G. Kumar, K. Narayan, "Review of non-reactive and reactive wetting of liquids on surfaces", Adv Colloid Interface Sci, Vol. 133, p. 61-89, 2007. DOI:10.1016/j.cis.2007.04.009

14. K. Narayan, P. Fernandes, "Determination of wetting behavior, spread activation energy, and quench severity of bioquenchants", Metall Mater Trans B, Vol. 38, p. 631-640, 2007. DOI:10.1007/s11663-007-9060-3

15. J.C. Halpin Tsai. "Primer on Composite Materials Analysis”, 2da Edición. p.165-191, 1992. 
16. E. Bedolla, J. Lemus-Ruiz, A. Contreras, "Synthesis and characterization of Mg-AZ91/AlN composite”, Material and Design, Vol. 38, p. 91-98, 2012. DOI:10.1016/j.matdes.2012.02.001

17. D.J. Lloyd, "Particle reinforcement aluminum and magnesium matrix composites", Intern. Mater. Rev., Vol. 39, No.1, p. 1-23, 1994. DOI: 10.1179/imr.1994.39.1.1

18. A.D. McLeod, C.M. Gabryel, "Kinetics of growth of spinel $\mathrm{MgAl}_{2} \mathrm{O}_{4}$ on alumina particulate in aluminum alloys containing magnesium”, Metall. Trans. A, Vol. 23, p. 1279-1283, 1992. DOI: 10.1007/BF02665059

19. D.J. Lloyd, H.P. Lagacé, A.D. McLeod, "Interfacial phenomena in metal matrix composites: Controlled interfaces in composite materials”, Edited by Hatsuo Ishida, Elsevier Science Publishing Co., Inc. 1990.

20. S.W. Lai, D.D. Chung, "Fabrication of particulate aluminum matrix composites by liquid metal infiltration". J. Mater. Sci., Vol. 29, p. 3128-3150, 1994. DOI: 10.1007/BF00356655

21. Q. Zhang, G. Chen, G. Wu, Z. Xiu, B. Luan, "Property characteristics of AlN/Al composite fabricated by squeeze casting technology". Mat. Lett., Vol. 57, p. 1453-1458, 2003. DOI:10.1016/S0167-577X(02)01006-6 